\title{
Application of statistical test on determining the unstable points in the basic network of horizontal displacement monitoring
}

\author{
Khanh Quoc Pham * \\ Hanoi University of Mining and Geology, Hanoi, Vietnam
}

\begin{abstract}
ARTICLE INFO
ABSTRACT

Article history:

Received $18^{\text {th }}$ Sept. 2020

Revised 09th Jan. 2021

Accepted 02 ${ }^{\text {nd }}$ Feb. 2021

Keywords:

Basis points,

Deformation monitoring, Horizontal displacement, Statistical testing.

The paper represents the hypothesis test method that can determine the instability control points of the reference network in the displacement of construction. Regarding data processing in displacement monitoring, the detection and modification for instability points is an important task because this affects the computation of the displacement of monitoring points. This method has been applied in many countries over the world but not in Vietnam, and it is processed through two steps including the global statistics test and local statistics test. The global statistics test is to identify whether a control point is stable or not. The local statistics test based on the division of groups is to find the unstable control points exactly. Experimental computation is carried out in two monitoring cycles at Hoa Binh hydroelectric plant. In this experiment, this algorithm detected two unstable points among six control points. This result is in agreement with the result that is solved by Vietnam's construction standard of TCVN 9399:2012. In conclusion, the hypothesis test method completely can apply in real geodetic production in Vietnam.
\end{abstract}

Copyright (C) 2021 Hanoi University of Mining and Geology. All rights reserved.

${ }^{*}$ Corresponding author

E - mail: phamquockhanh@humg.edu.vn

DOI: 10.46326/JMES.2021.62(1).05 


\title{
Tạp chí Khoa học Kỹ thuật Mỏ - Địa chất
}

Trang điện tử: http://tapchi.humg.edu.vn

\section{Ứng dụng kiểm định thống kê xác định điểm không ổn định trong lưới cơ sở quan trắc chuyển dịch biến dạng công trình}

\author{
Phạm Quốc Khánh * \\ Trường đại học Mỏ - Địa chất, Hà Nội, Việt Nam
}

THÔNG TIN BÀI BÁO

Quá trình:

Nhận bài 18/9/2020

Sửa xong 09/01/2021

Chap nhận đăng 02/02/2021

Tù khóa:

Chuyển dịch ngang,

Điểm cơ' sở,

Kiểm định thống kê,

Quan trắc biến dạng.
TÓM TẮT

Bài báo giới thiệu một phương pháp tìm điểm không ổn định của lưới cơ sở trong quan trắc chuyển dịch ngang công trình dựa trên thuật toán kiểm định thống kê. Đối với công tác xư lý số liệu lưới quan trắc chuyển dịch ngang công trình, việc xác định và hiệu chỉnh điểm lưới cơ sở không ổn định là một bước rất quan trọng, không thể thiếu vì nó quyết định đến việc tính toán lượng chuyển dịch của các điểm quan trắc. Phương pháp này hiện đang được sử dụng ở nhiều nước trên thế giới nhưng hiện chưa được áp dụng ở Việt Nam, và được thực hiện dựa trên hai bước co bản gồm kiểm nghiệm tổng quát và kiểm nghiệm cục bộ. Kiểm nghiệm tổng quát là để xác định xem mạng lưới có điểm không ổn định hay không. Kiểm đinh cuc bộ dựa trên việc chia nhóm để tìm ra điểm không ổn định trong lưới. Tính toán thực nghiệm được thực hiện cho hai chu kỳ đo lưới cơ sở quan trắc chuyển dịch ngang Thủy điện Hòa Bình. Thuật toán đã xác định được hai điểm không ổn định trong tổng số sáu điểm của lưới. Kết quả này hoàn toàn thống nhất với phương pháp phân tích độ ổn định của mốc lưới cơ sở theo tiêu chuẩn TCVN9399: 2012. Qua đó cho thấy, hoàn toàn có thể úng dụng phương pháp phân tích độ ổn định các mốc lưới dựa trên bài toán kiểm định thống kê trong thực tế sản xuất trắc địa ở Việt Nam.

(C) 2021 Trường Đại học Mỏ - Địa chất. Tất cả các quyền được bảo đảm.

\section{Mở đầu}

Các điểm cơ sở của lưới quan trắc chuyển dịch biến dạng nếu bị dịch chuyển trong quá trình sử dụng sẽ ảnh hưởng trực tiếp đến việc tính toán lượng chuyển dịch của các điểm quan trắc. Từ đó, ảnh hưởng tới kết quả phân tích chuyển dịch của

\footnotetext{
*Tác giả liên hệ

E - mail: phamquockhanh@humg.edu.vn

DOI: 10.46326/JMES.2021.62(1).05
}

đối tượng quan trắc, dẫn đến nhận định và kết luận không đúng. Làm thế nào để có thể phát hiện và xác định đúng điểm lưới cơ sở bị chuyển dịch là bài toán được các nhà trắc địa trong và ngoài nước nghiên cứu từ lâu. ở Việt Nam, thường ứng dụng bài toán bình sai lưới tự do với thuật toán tính lặp để phân tích độ ổn định điểm lưới cơ sở, sau đó lấy kết quả bình sai khi loại bỏ điểm không ổn định để so sánh với tọa độ các điểm chu kỳ 1 và chu kỳ trước đó (Trần Khánh và nnk., 2014; Trần Khánh, 2010; Nguyễn Quang Phúc, Hoàng Anh Thế, 2009). Hiện nay, ở một số nước sử dụng nhiều phương pháp xác định điểm lưới không ổn định, 
có thể kể đến: phương pháp độ lệch giới hạn, phương pháp thay thế lặp, phương pháp phân lượng chuyển dịch điểm đơn (Tao Benzao, 2001; Huang Shengxiang và nnk., 2013; Hou Jianguo, Wang Tengjun, 2008; Huang Shengxiang, 2001); phương pháp tổ hợp phương sai hậu nghiệm (Phạm Quốc Khánh, Zhang Zhenglu, 2013), phương pháp phân tích dựa vào tín hiệu GPS (Amiri-Simkooei, 2016). Nhưng nổi bật và được ứng dụng nhiều hơn cả là ứng dụng kiểm định thống kê (còn có tên gọi khác là phương pháp chênh lệch trung bình) do Pelzer đề xuất (Huang Shengxiang và nnk., 2013). Phương pháp này sử dụng kiểm định thống kê nên có cơ sở khoa học chặt chẽ, thuật toán rõ ràng và dễ lập trình tự động hóa. Chính vì vậy, bài báo này nghiên cứu lý thuyết và ứng dụng kiểm định thống kê xác định điểm không ổn định của lưới cơ sở quan trắc chuyển dịch biến dạng công trình. Kết quả phân tích từ lưới cơ sở quan trắc Thủy điện Hòa Bình chứng tỏ phương pháp này cho kết quả chính xác và tin cậy.

\section{Nội dung phương pháp}

Ứng dụng kiểm định thống kê xác định điểm không ổn định của lưới cơ sở quan trắc chuyển dịch biến dạng công trình được thực hiện qua hai bước. Một là kiểm định tính thống nhất đồ hình lưới của hai chu kỳ cần phân tích, gọi là kiểm nghiệm tổng quát, nếu lượng thống kê sau kiểm định được chấp nhận thì xác nhận rằng tất cả các điểm lưới cơ sở của chu kỳ đang xét đều ổn định, không cần thực hiện bước thứ hai. Ngược lại, kiểm nghiệm bị bác bỏ thì cần thông qua bước thứ hai là kiểm nghiệm cục bộ, thông qua kiểm nghiệm lượng thống kê được thành lập khi đã loại trừ điểm có mức độ chuyển dịch từ cao đến thấp bằng phương pháp tính lặp, khi lượng thống kê được chấp nhận thì dừng (Huang Shengxiang và nnk., 2013).

\subsection{Kiểm nghiệm tổng quát}

Giả thiết i và $\mathrm{j}$ là hai chu kỳ quan trắc ở hai thời điểm khác nhau $(i<j)$, để có thể xác định tính thống nhất đồ hình giữa hai chu kỳ cần tiến hành kiểm nghiệm tổng quát. Dựa vào kết quả bình sai lưới tự do với số khuyết dương của từng chu kỳ với hệ tham khảo của lưới như nhau, phương sai sau bình sai của từng chu kỳ được tính:

$$
\begin{aligned}
\mu_{i}^{2} & =\frac{\left(V^{T} P V\right)^{i}}{f_{i}} \\
\mu_{j}^{2} & =\frac{\left(V^{T} P V\right)^{j}}{f_{j}}
\end{aligned}
$$

Trong đó: $V, P$ - vector số hiệu chỉnh và trọng số của trị đo tương ứng với chu kì $i$ và chu kỳ $j, f_{i}$ và $f_{j}$ - số trị đo thừa của chu kỳ $i$ và chu kỳ $j$. Thông thường độ chính xác của hai chu kỳ khác nhau là tương đương vì cùng đồ hình, máy móc, người đo,... nhưng do ảnh hưởng của sai số nên phương sai ước lượng không thể như nhau. Khi đó, cần kiểm nghiệm độ chính xác tương đồng của hai chu kỳ thì mới có thể so sánh độ lệch của chúng. Phương sai tổng hợp được tính:

$$
\mu^{2}=\frac{\left(V^{T} P V\right)^{i}+\left(V^{T} P V\right)^{j}}{f}
$$

Trong đó: $f=f_{i}+f_{j}$, - tổng số trị đo thừa của hai chu kỳ đo.

Dựa vào kết quả sau bình sai của hai chu kỳ quan trắc lưới cơ sở, sẽ tính được véc tơ hiệu tọa độ giữa hai chu kỳ (khoảng chênh lệch tọa độ) là:

$$
d=\widehat{X}_{J}-\widehat{X}_{l}
$$

Trong đó: $\widehat{X}_{J}, \widehat{X}_{l}$ - véc tơ tham số tương ứng của từng chu kỳ sau bình sai.

Ma trận hiệp trọng số đảo của khoảng chênh lệch $d$ là:

$$
Q_{d}=Q_{i}+Q_{j}
$$

Trong đó: $Q_{i}, Q_{j}$ - ma trận hiệp trọng số đảo của các tham số tương ứng của chu kỳ 1 và chu kỳ $j$. Phương sai của khoảng chênh lệch $d$ được tính

$$
\mu_{i}^{2}=\frac{d^{T} Q_{d}^{+} d}{f_{d}} \quad \mu_{i}^{2}=\frac{d^{T} P_{d} d}{f_{d}}
$$

Trong đó: $P_{d}=Q_{d}^{+}$- ma trận nghịch đảo tổng quát của 2 ma trận $P_{d}+Q_{j}, f_{d}$ - số lượng số hiệu chỉnh tọa độ độc lập của vector chênh lệch $d$, cũng chính là số ẩn số trong lưới.

Dùng phép kiểm định Fisher (viết tắt là kiểm định $F$ ), lập lượng thống kê.

$$
T=\frac{\mu_{d}^{2}}{\mu^{2}} \sim F\left(\alpha ; f_{d}, f\right)
$$

Với giả thiết gốc $H_{0}$ là vị trí các điểm lưới cơ sở giữa 2 chu kỳ quan trắc đều ổn định. Khi đó, lượng thống kê $T$ tuân theo luật phân phối $F$ với 
bậc tự do là $\left(f_{d}, f\right)$ với mức tin cậy $\alpha$ lấy bằng 0,05 hoặc 0,01 . Tra bảng xác xuất để có được phân vị tương ứng. Nếu $T \leq F\left(\alpha, f_{d}, f\right)$ thì chấp nhận giả thiết gốc, tức là tất cả các điểm của lưới cơ sở đều ổn định; ngược lại, bác bỏ giả thiết gốc, kiểm nghiệm không được thông qua. Nghĩa là trong lưới có điểm không ổn định, cần tìm và loại trừ điểm không ổn định này.

Để có thể xác định được điểm lưới cơ sở nào không ổn định, cần tiến hành kiểm nghiệm cục bộ mới có thể kết luận chính xác.

\subsection{Kiểm nghiệm cục bộ}

Từ vector khoảng chênh lệch $d$, giả thiết điểm lưới cơ sở được chia thành hai nhóm là nhóm các điểm ổn định mang chỉ số $F$ và nhóm các điểm chuyển dịch mang chỉ số $M$. Khi đó có thể tách vector chênh lệch và ma trận hiệp trọng số đảo của nó thành:

$$
d=\left[\frac{d_{F}}{d_{M}}\right], Q_{d}^{+}=\left[\begin{array}{ll}
P_{F F} & P_{F M} \\
P_{M F} & P_{M M}
\end{array}\right]
$$

Trong đó, chỉ số $M$ trong lần lặp thứ nhất biểu thị một điểm chuyển dịch $i$, chỉ số $F$ biểu thị các điểm khác.

Thực hiện biến đổi như sau (Huang Shengxiang và nnk., 2013).

$$
\left\{\begin{array}{c}
\bar{d}_{M}=d_{M}+P_{M M}^{-1} P_{M F} d_{F} \\
\bar{P}_{F F}=P_{F F}+P_{F M} P_{M M}^{-1} P_{M F}
\end{array}\right.
$$

Và phân tích $d^{T} Q_{d}^{+} d$ thành:

$$
d^{T} Q_{d}^{+} d=d_{F F}^{T} \bar{P}_{F F} d_{F}+\bar{d}_{M M}^{T} P_{M M} \bar{d}_{M}
$$

Khi bắt đầu tính toán, giả định trong lưới chỉ có 1 điểm i chuyển dịch, lần lượt tính giá trị $\left(\bar{d}_{M M}^{T} P_{M M} \bar{d}_{M}\right)_{i} \quad$ với $i=1,2, \ldots, t$. Sau đó, lấy $\max \left(\bar{d}_{M M}^{T} P_{M M} \bar{d}_{M}\right)_{i}$ là điểm nghi ngờ bị chuyển dịch, loại bỏ điểm này, tiến hành lặp lại quá trình trên với các điểm còn lại, khi đó sẽ lập được lượng thống kê:

$$
T_{1}=\frac{\mu_{d f}^{2}}{\mu^{2}} \sim F\left(\alpha ; f_{d f}, f\right)
$$

Trong đó: $\mu_{d f}^{2}=\frac{d_{F}^{T} \bar{P}_{F F} d_{F}}{f_{d f}}, f_{d f}=f_{d}-1$. Khi $T_{1} \leq F\left(\alpha, f_{d f}, f\right)$ nghĩa là các điểm còn lại trong lưới đều ổn định, quá trình phân tích độ ổn định kết thúc. Ngược lại, sẽ loại bỏ tiếp điểm bị chuyển dịch và lại tiến hành kiểm nghiệm. Quá trình này lặp lại cho đến khi giả thiết gốc được chấp nhận thì có thể kết luận các điểm lưới còn lại là các điểm ổn đinhn.

\section{Tính toán thực nghiệm}

Bài báo sử dụng lưới cơ sở quan trắc chuyển dịch ngang Thủy điện Hòa Bình làm thực nghiệm. Đây là thủy điện lớn thứ 2 của nước ta sau Thủy điện Sơn La, được xây dựng là tuyến dạng vòm. Lưới cơ sở quan Trắc Thủy điện Hòa Bình được xây dựng ở phía hạ lưu gồm 6 mốc chuẩn ký hiệu là T4, T13, T16, T17, M12 và M15 như Hình 1. Ở mỗi chu kỳ quan trắc, lưới này được đo bằng máy toàn đạc điện tử độ chính xác cao của Thụy Sĩ là TC2003, độ chính xác đo góc theo lý lịch máy là 1", độ chính xác đo cạnh là $1+1$ ppm. Để có thể phân tích và xác định điểm lưới không ổn định, cần phải có ít nhất hai chu kỳ đo.

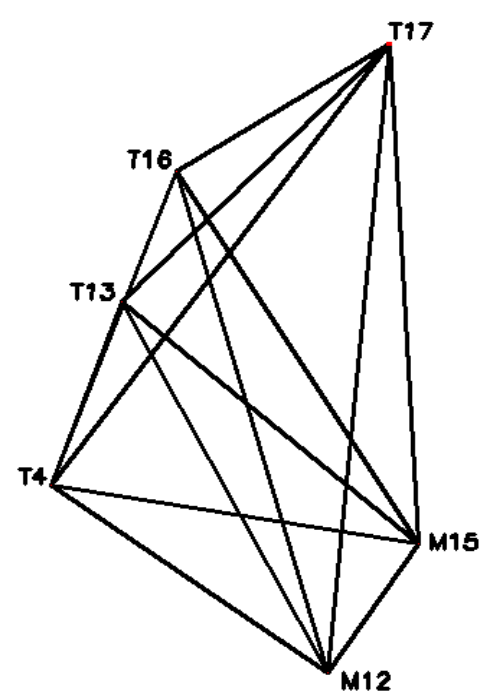

Hình 1. Sơ đồ lưới cơ sở quan trắc chuyển dịch ngang Thủy điện Hòa Bình.

Lưới cơ sở của Thủy điện Hòa Bình được quan trắc định kỳ, trong Bảng 1 là số liệu đo lưới của hai chu kỳ, ký hiệu là chu kỳ $i$ và $j(i<j)$. Mạng lưới cơ sở đo tất cả các cạnh, tổng số có 14 cạnh, lưới được bình sai theo phương pháp lưới tự do với số khuyết dương.

Bảng 1. Số liệu đo khoảng cách các điểm cơ sở quan trắc chuyển dịch ngang Thủy điện Hòa Bình.

\begin{tabular}{|r|r|r|r|r|}
\hline \multirow{2}{*}{ TT } & \multicolumn{2}{|c|}{ Tên cạnh } & \multicolumn{2}{c|}{ Giá trị cạnh $(\mathrm{m})$} \\
\cline { 2 - 5 } & Đ_đầu & Đ_Cuối & \multicolumn{1}{c|}{ Chu kỳ $i$} & \multicolumn{1}{c|}{ Chu kỳ $j$} \\
\hline 1 & T16 & T17 & 611,5485 & 611,5525 \\
\hline 2 & T16 & T4 & 875,626 & 875,623 \\
\hline 3 & T16 & M12 & 1361,074 & 1361,069 \\
\hline
\end{tabular}




\begin{tabular}{|c|r|r|r|r|}
\hline 4 & T16 & M15 & 1135,527 & 1135,520 \\
\hline 5 & T17 & T13 & 931,8665 & 931,866 \\
\hline 6 & T17 & T4 & 1413,048 & 1413,048 \\
\hline 7 & T17 & M12 & 1650,317 & 1650,315 \\
\hline 8 & T17 & M15 & 1307,279 & 1307,277 \\
\hline 9 & M15 & T13 & 954,867 & 954,861 \\
\hline 10 & M15 & M12 & 403,951 & 403,949 \\
\hline 11 & M15 & T4 & 899,748 & 899,744 \\
\hline 12 & T13 & T4 & 510,3085 & 510,3065 \\
\hline 13 & T13 & M12 & 1089,186 & 1089,181 \\
\hline 14 & T4 & M12 & 826,6125 & 826,6095 \\
\hline
\end{tabular}

Bảng 2 là tọa độ gần đúng của các điểm lưới. Sau bình sai, tọa độ thu được ghi trong Bảng 3.

Bảng 2. Bảng tọa độ gần đúng.

\begin{tabular}{|c|c|c|}
\hline \multirow{2}{*}{ Tên điểm } & \multicolumn{2}{|c|}{ Tọa độ gần đúng $(\mathrm{m})$} \\
\cline { 2 - 3 } & $X$ & $Y$ \\
\hline T4 & 2235,538 & 3675,617 \\
\hline M12 & 1746,337 & 4341,923 \\
\hline T13 & 2716,359 & 3846,571 \\
\hline M15 & 2084,667 & 4562,620 \\
\hline T16 & 3057,607 & 3977,141 \\
\hline T17 & 3389,950 & 4490,504 \\
\hline
\end{tabular}

Bảng 3. Bảng tọa độ sau bình sai của 2 chu kỳ.

\begin{tabular}{|c|c|c|c|c|}
\hline \multirow{2}{*}{$\begin{array}{c}\text { Tên } \\
\text { điểm }\end{array}$} & \multicolumn{2}{|c|}{$\begin{array}{c}\text { Tọa độ sau bình sai } \\
\text { chu kỳ } i(\mathrm{~m})\end{array}$} & \multicolumn{2}{c|}{$\begin{array}{c}\text { Tọa độ sau bình sai } \\
\text { chu kỳ } j(\mathrm{~m})\end{array}$} \\
\cline { 2 - 5 } & $X$ & $Y$ & $X$ & $Y$ \\
\hline T4 & 2235,5388 & 3675,6159 & 235,5390 & 3675,6169 \\
\hline M12 & 1746,3332 & 4341,9235 & 746,3358 & 341,9230 \\
\hline T13 & 2716,3596 & 3846,5707 & 716,3580 & 3846,5729 \\
\hline M15 & 2084,6637 & 4562,6238 & 2084,6658 & 4562,6207 \\
\hline T16 & 3057,6125 & 3977,1388 & 3057,6091 & 3977,1380 \\
\hline T17 & 3389,9503 & 4490,5034 & 3389,9504 & 4490,5045 \\
\hline
\end{tabular}

Phương sai tương ứng sau bình sai của chu kỳ $i$ và chu kỳ $j$ là:

$$
\begin{aligned}
\mu_{i}^{2} & =\frac{\left(V^{T} P V\right)^{1}}{f_{j}}=0,3364 \\
\mu_{j}^{2} & =\frac{\left(V^{T} P V\right)^{j}}{f_{j}}=0,4356
\end{aligned}
$$

\subsection{Kiểm nghiệm tổng quát}

Sau khi bình sai lưới cơ sở trên theo phương pháp tự do với cùng tọa độ gần đúng của các điểm tương ứng, tính được lượng chuyển dịch của điểm lưới cơ sở giữa 2 chu kỳ:

$$
\begin{gathered}
d=(0.21,02,6-0,5-1,62,22,1 \\
-3,1-3,4 \\
-0,80,11,1)^{T}
\end{gathered}
$$

Từ đó tính được phương sai của khoảng chênh lệch $d$ :

$$
\mu_{d}^{2}=\frac{d^{T} Q_{d}^{+} d}{f_{d}}=\frac{25,36}{12}=2,11
$$

Sai số trung phương trọng số đơn vị tổng hợp của 2 chu kỳ:

$$
\mu^{2}=\frac{\left(V^{T} P V\right)^{i}+\left(V^{T} P V\right)^{j}}{f}=0,39
$$

Thành lập lượng thống kê:

$$
T=\frac{\mu_{d}^{2}}{\mu^{2}}=\frac{2,11}{0,39}=5,47
$$

Do $T=5,47>F(0,05 ; 12,10)=1,61$ nên nghi ngờ lưới cơ sở quan trắc Thủy điện Hòa Bình có điểm bị chuyển dịch.

\subsection{Kiểm nghiệm cục bộ}

Để tiến hành xác định điểm nào trong lưới là điểm bị chuyển dịch, đầu tiên tính lượng chuyển dịch tổng thể của các điểm lưới, thu được kết quả như Bảng 4.

Bảng 4. Chuyển dịch tổng hợp của các điểm lưới.

\begin{tabular}{|c|c|c|c|c|}
\hline \multirow{2}{*}{ TT } & \multirow{2}{*}{ Tên điểm } & \multicolumn{3}{|c|}{ Lượng chuyển dịch $(\mathrm{mm})$} \\
\cline { 3 - 5 } & & $\Delta X$ & $\Delta Y$ & $m_{p}$ \\
\hline 1 & T4 & 0,2 & 1,0 & 1,0 \\
\hline 2 & M12 & 2,6 & $-0,5$ & 2,6 \\
\hline 3 & T13 & $-1,6$ & 2,2 & 2,7 \\
\hline 4 & M15 & 2,1 & $-3,1$ & 3,7 \\
\hline 5 & T16 & $-3,4$ & $-0,8$ & 3,5 \\
\hline 6 & T17 & 0,1 & 1,1 & 1,1 \\
\hline
\end{tabular}

Lấy điểm có độ chuyển dịch lớn nhất (điểm M15) tiến hành kiểm nghiệm, theo công thức (7) đến (9) tính được lượng thống kê:

$$
\begin{gathered}
T_{1}=\frac{\mu_{d f_{1}}^{2}}{\mu^{2}}=\frac{1,78}{0,39}=4,6>F(0,05 ; 11,10) \\
=1,61
\end{gathered}
$$

Do $T_{1}>F$ nên giả thiết gốc bị bác bỏ, chứng tỏ điểm M15 bị chuyển dịch. Loại bỏ điểm M15, với các điểm còn lại, điểm T16 là điểm có lượng 
chuyển dịch lớn nhất, lặp lại cách tính trên thu được lượng thống kê:

$$
\begin{aligned}
T_{2}=\frac{\mu_{d f_{2}}^{2}}{\mu^{2}}=\frac{1,36}{0,39}=3,53 \\
\quad>F(0,05 ; 10,10)=1,61
\end{aligned}
$$

Kết quả thu được cho thấy điểm T16 cũng là điểm bị chuyển dịch, tiếp tục phép lặp với điểm T13, lượng thống kê:

$$
\begin{aligned}
T_{3}=\frac{\mu_{d f_{3}}^{2}}{\mu^{2}}=\frac{0,00052}{0,39}=1,34 \\
\quad<F(0,05 ; 9,10)=1,61
\end{aligned}
$$

Vì $T_{3}<F(0,05 ; 9,10)=1,61$ nên giả thiết gốc được chấp nhận, tức điểm cơ sở T13 được coi là ổn định. Điều đó cũng có nghĩa là các điểm còn lại trong lưới là điểm T4, M12, T17 đều được coi là ổn định.

Nếu chỉ lấy tọa độ các điểm ổn định để bình sai lưới cơ sở, tọa độ sau bình sai của các điểm lưới và lượng chuyển dịch của chúng được ghi trong Bảng 5 và Bảng 6 .

Bảng 5. Tọa độ điểm lưới sau bình sai chu kỳ 2 sau khi loại bỏ điểm không ổn định.

\begin{tabular}{|c|c|c|c|c|c|}
\hline \multirow{2}{*}{ TT } & \multirow{2}{*}{$\begin{array}{l}\text { Tên } \\
\text { điểm }\end{array}$} & \multicolumn{3}{|c|}{ Lượng chuyển dịch (mm) } & \multirow[t]{2}{*}{ Ghi chú } \\
\hline & & $\Delta X$ & $\Delta Y$ & $m_{p}$ & \\
\hline 1 & $\mathrm{~T} 4$ & 0,15 & 0,59 & 0,61 & \\
\hline 2 & M12 & 2,94 & $-0,61$ & 3,00 & \\
\hline 3 & T13 & $-1,53$ & 1,52 & 2,15 & \\
\hline 4 & M15 & 2,55 & $-3,36$ & 4,22 & $\begin{array}{l}\text { Không ổn } \\
\text { định }\end{array}$ \\
\hline 5 & T16 & $-3,21$ & $-1,62$ & 3,59 & $\begin{array}{l}\text { Không ổn } \\
\text { đinh }\end{array}$ \\
\hline 6 & T17 & 0,54 & 0,01 & 0,54 & \\
\hline
\end{tabular}

\begin{tabular}{|c|c|c|c|c|}
\multirow{2}{*}{ TT } & \multirow{2}{*}{$\begin{array}{c}\text { Tên } \\
\text { điểm }\end{array}$} & \multicolumn{2}{|c|}{ Tọa độ sau bình sai chu kỳ j (m) } & Ghi chú \\
\cline { 4 - 5 } & T4 & 2235,5389 & 675,6165 & \\
\hline 2 & M12 & 1746,3361 & 341,9229 & \\
\hline 3 & T13 & 2716,3581 & 846,5722 & \\
\hline 4 & M15 & 2084,6662 & 562,6204 & $\begin{array}{c}\text { Không } \\
\text { ôn định }\end{array}$ \\
\hline 5 & T16 & 3057,6093 & 977,1372 & $\begin{array}{c}\text { Không } \\
\text { ổn định }\end{array}$ \\
\hline 6 & T17 & 3389,9508 & 490,5034 & \\
\hline
\end{tabular}

Bảng 6. Lượng chuyển dịch của các điểm sau phân tích.
Nhận xét: Phương pháp kiểm định thống kê thông qua thành lập lượng thống kê, kiểm nghiệm để phân tích độ ổn định điểm lưới cơ sở Thủy điện Hòa Bình của chu kỳ j so với chu kỳ $i$ đã phát hiện 2 điểm không ổn định là điểm M15 và T16, các điểm cơ sở còn lại đều ổn định, có thể dùng làm gốc cho mang lưới quan trắc.

Để có cơ sở kết luận về tính chính xác của phương pháp nghiên cứu, bài báo sử dụng số liệu đo của 2 chu kỳ lưới cơ sở Thủy điện Hòa Bình như trong Bảng 3, tiến hành phân tích độ ổn định của lưới theo (Trần Khánh, Nguyễn Quang Phúc, 2010). Với tiêu chuẩn độ ổn định của lưới là $3 \mathrm{~mm}$, sau hai lần lặp cũng thu được kết quả như Bảng 6 . Điều đó chứng tỏ phương pháp này có đủ cơ sở về độ chính xác và độ tin cậy.

\section{Kết luận}

- Phương pháp ứng dụng xác xuất thống kê tìm điểm không ổn định trong lưới quan trắc chuyển dịch biến dạng công trình có lý thuyết chặt chẽ về mặt toán học, có độ tin cậy tốt.

- Kết quả tìm kiếm điểm không ổn định lưới cơ sở quan trắc chuyển dịch ngang Thủy điện Hòa Bình là chính xác, thông qua lượng thống kê thành lập cho các điểm có lượng chuyển dịch từ lớn đến nhỏ cho phép xác định được điểm không ổn định.

- Phương pháp này hoàn toàn có thể áp dụng trong thực tế sản xuất khi xử lý số liệu lưới cơ sở quan trắc công trình.

\section{Tài liệu tham khảo}

TCVN9399:2012- Nhà và công trình xây dựng, xác định chuyển dịch ngang bằng phương pháp trắc địa.

Amiri-Simkooei A. R., Alaei - Tabatabaei, S. M, Zangeneh-Nejad, F., \& Voosoghi, B. (2017). Stability analysis of deformation-monitoring network points using simultaneous observation adjustment of two epochs. Journal of Surveying Engineering, 143(1), 04016020.

Hoang Shengxiang. (2001). Phân tích tính ổn định lưới quan trắc. Tạp chí công trình và thông tin Trắc Hội, 3, 16-19, tiếng Trung Quốc

Hou Jianguo, Wang Tengjun. (2008). Lý thuyết và úng dụng quan trắc biến dạng. Nhà xuất bản Trắc hội Bắc Kinh, tiếng Trung Quốc. 
Huang Shengxiang, Yin Hui, Jiang Zheng, (2013). Xử lý số liệu quan trắc biến dạng. Nhà xuất bản Đại học Vũ Hán, tiếng Trung Quốc.

Nguyễn Quang Phúc, Hoàng Anh Thế, (2009). Nghiên cứu phương pháp phân tích độ ổn định các mốc lưới cơ sở quan trắc chuyển dịch ngang đo bằng công nghệ GPS. Tạp chí Khoa học kỹ thuật Mỏ - Địa chất, 26, 83-86.

Phạm Quốc Khánh, Zhang Zhenglu. (2013). Một phương pháp xác định độ ổn định điểm lưới cơ sở trong quan trắc biến dạng công trình đường hầm. Hội nghi khoa học Viện khoa học công nghệ xây dựng, 207-210.
Tao Benzao. (2001). Bình sai lưới tự do và phân tích biến dạng. Nhà xuất bản Đại học Khoa học Trắc hội Vũ Hán, tiếng Trung Quốc.

Trần Khánh. (2010). Phân tích độ ổn định hệ thống mốc độ cao cơ sở trong quan trắc lún công trình. Tạp chí Cầu đường Việt Nam, 5.

Trần Khánh, Lê Đức Tinh, Nguyễn Hà. (2014). Phân tích độ ổn định lưới cơ sở quan trắc chuyển dịch ngang công trình theo thuật toán bình sai hiệu trị đo. Tạp chí Khoa học Kỹ thuật Mỏ - Địa chất, 45.

Trần Khánh, Nguyễn Quang Phúc. (2010). Quan trắc chuyển dịch và biến dạng công trình. Nhà xuất bản Giao thông vận tải. 